\title{
Efectos del metabisulfito de sodio sobre la actividad y composición proteásica de los extractos enzimáticos del langostino Pleoticus muelleri
}

\author{
Clara Liebana ${ }^{1,2}$, Analía V. Fernández-Giménez ${ }^{1,2}$ y Nair de los Ángeles Pereira ${ }^{1,2,3, *}$ \\ ${ }^{1}$ Departamento de Ciencias Marinas, Facultad de Ciencias Exactas y Naturales, Universidad Nacional de Mar del Plata (UNMdP), \\ Funes 3350, B7602AYL - Mar del Plata, Argentina. ${ }^{2}$ Instituto de Investigaciones Marinas y Costeras (IIMyC-CONICET), \\ Facultad de Ciencias Exactas y Naturales, Universidad Nacional de Mar del Plata (UNMdP), Consejo Nacional de Investigaciones Científicas \\ y Técnicas (CONICET), CC 1260. 7600 - Mar del Plata, Argentina. ${ }^{3}$ Universidad Tecnológica Nacional (UTN), Regional Mar del Plata, \\ Buque Pesquero Dorrego 281, B7600CLF - Mar del Plata, Argentina. ORCID Clara Liebana (D) https://orcid.org/0000-0002-7062-8675, \\ Analía V. Fernández-Giménez (D) https://orcid.org/0000-0001-9232-4560, Nair de los Ángeles Pereira (D) https://orcid.org/0000-0002-7341-5333
}

Marine and Fishery Sciences MAFIS 


\section{INTRODUCCIÓN}

La pesquería del langostino, Pleoticus muelleri, una de las actividades económicas más importantes del país, presentó en los últimos cinco años un incremento en las cifras de captura, alcanzando en 2020 un total de $183.892,9$ t desembarcadas y procesadas (MAGyP 2021). Este procesamiento en tierra genera una gran cantidad de residuos sólidos, conformados principalmente por cabezas, que representan el 50\% del volumen total. Parte de estos desechos suele ser enterrada en basurales a cielo abierto sin tratamiento adecuado, mientras que otra parte es enterrada mediante el sistema landfarming; sin embargo, la contratación de este servicio significa un alto costo para la industria pesquera (Pereira y Fernández-Giménez 2016).

Por otro lado, los mercados internacionales exigen un producto con carne de consistencia firme, exoesqueleto rígido y sin presencia de melanosis o black spot. Este último proceso ocurre luego de la muerte del organismo, donde se genera una coloración negruzca causada por el complejo enzimático polifenoloxidasa (PFO). La enzima reacciona con el contenido celular oxidando los fenoles a quinonas en presencia de oxígeno molecular, formando pigmentos insolubles que se desarrollan principalmente en un medio alcalino (Díaz-López et al. 2003; Carranza-Espinal 2014). El almacenamiento en hielo o el congelado de los langostinos, puede reducir su actividad enzimática, pero no detenerla, por lo que la PFO sigue actuando lentamente a temperaturas de refrigeración y puede acelerarse durante el descongelado. Si bien la melanosis no influye sobre el sabor y el aroma, es un signo de mal manejo del producto y baja calidad. Estos langostinos son rechazados por los consumidores, ya que el oscurecimiento se asocia a la putrefacción, pudiendo causar importantes pérdidas económicas debido al alto valor que tienen estos crustáceos en el mercado (Díaz-López et al. 2003; Carranza-Espinal 2014; Xu et al. 2018).
El metabisulfito de sodio (MBS) es el compuesto químico más utilizado para controlar la melanosis de los langostinos durante el almacenamiento (Carranza-Espinal 2014). Este preservante actúa inhibiendo o retardando el proceso de oscurecimiento mediante dos mecanismos: a) reaccionando con quinonas intermedias producidas en la reacción de melanosis, evitando su polimerización en compuestos pigmentados y formando sulfoquinonas, y b) inhibiendo irreversiblemente la PFO, probablemente por la ruptura de enlaces disulfuro (Ferrer et al. 1989). La metodología comúnmente utilizada para preservar la apariencia y calidad del langostino consiste en el baño de inmersión en solución de MBS. Luego de su captura, los langostinos son colocados en recipientes con una solución acuosa de MBS al 3\% a temperaturas por debajo de $5{ }^{\circ} \mathrm{C}$ durante $30 \mathrm{seg}$. Luego, se almacenan en hielo con MBS al $0,5 \%$ hasta su procesamiento. De esta manera, desde el momento de la pesca hasta el empaque, el langostino absorbe MBS durante 6 a $12 \mathrm{~h}$, alcanzando concentraciones de hasta 100 ppm de MBS en el tejido al momento de su consumo, niveles que se corresponden con los permitidos por el Consejo de la Unión Europea y la Food and Drug Administration (Otell 2010). Posteriormente al tratamiento, los langostinos son separados por tamaño para su comercialización, generándose dos tipos de desechos: cabezas y langostinos enteros (rotos o de menor tamaño al comercializado) (Pettovello y Boschi 2016; Moriondo-Danovaro y de la Garza 2019). Por otro lado, existe una importante porción de langostinos que son capturados incidentalmente como fauna acompañante (descarte) de otras especies objetivo, principalmente de la merluza común (Merluccius hubbsi) (Góngora et al. 2012; Bovcon et al. 2013). Estos langostinos descartados, al no tener un fin comercial, no son tratados con MBS.

En trabajos previos se ha demostrado que $P$. muelleri es una fuente importante de biomoléculas y subproductos tales como suplementos enzimáticos para alimentos, coagulantes lácteos e hidroli- 
zados proteicos (Pereira y Fernández-Giménez 2016, 2017; Rodríguez et al. 2017, 2018; Pereira et al. 2018, 2020a, 2020b, 2021; Rodríguez et al. 2021). Es por ello que resulta fundamental contar con proteasas funcionales que presenten altos niveles de actividad enzimática. Sin embargo, el MBS utilizado para preservar el langostino comercial podría tener un efecto negativo sobre la actividad proteásica e interferir en la obtención de estos subproductos. El objetivo de este trabajo fue comparar la actividad enzimática y la composición de proteasas activas en las cabezas de $P$. muelleri (tratadas y sin tratar con MBS) para contar con información valiosa a la hora de proponer la valorización de estos residuos, en particular, el reaprovechamiento de las enzimas digestivas del langostino en procesos biotecnológicos.

\section{MATERIALES Y MÉTODOS}

\section{Obtención del material biológico}

En este trabajo se utilizaron 20 ejemplares de langostinos de talla comercial L1 (con un promedio de 20,25 $\mathrm{cm}$ de largo total y $70 \mathrm{~g}$ de peso total) provistos por la empresa PESCO de Mar del Plata, y procedentes de la pesca comercial de langostino en la zona del Golfo San Jorge. Estos ejemplares fueron tratados con MBS por la empresa pesquera luego de su captura, tal como se describió previamente (en adelante "tratados"). Como control se utilizó otro lote de 20 ejemplares L1 integrantes de la fauna acompañante de embarcaciones fresqueras dedicadas a la pesca de merluza en el Golfo San Jorge, sin preservante (en adelante "sin tratar").

\section{Preparación de extractos enzimáticos y cuanti- ficación de proteína soluble}

Se tomaron al azar 15 langostinos de cada lote (tratados y sin tratar con MBS) y se utilizaron las cabezas para preparar los extractos enzimáticos. Los homogenatos se realizaron en agua destilada $(1: 4 \mathrm{p} / \mathrm{v})$ y luego fueron centrifugados por $30 \mathrm{~min}$ a $4{ }^{\circ} \mathrm{C}$ y $10.000 \mathrm{~g}$ (Presvac EPF-12R, Argentina). Los lípidos fueron removidos, los sobrenadantes fueron almacenados a $-20{ }^{\circ} \mathrm{C}$ y utilizados como extractos enzimáticos para los ensayos posteriores. La concentración de proteína soluble se evaluó en todos los extractos enzimáticos de acuerdo con el método de Bradford (1976) utilizando seroalbúmina bovina como proteína estándar (Sigma A9647).

\section{Determinación de actividad de proteasas áci- das y alcalinas}

La actividad de proteasas ácidas fue determinada en los extractos enzimáticos (tratados y sin tratar con MBS) de acuerdo con la técnica de Anson (1938) utilizando hemoglobina bovina (Sigma $\mathrm{H} 2625)$ al 0,5\% (p/v) como sustrato, en buffer Glicina- $\mathrm{HCl} 0,1 \mathrm{M} \mathrm{pH} 3$. Las alícuotas de cada extracto proteico $(10 \mu \mathrm{l})$ fueron incubadas durante 10 min con sustrato disuelto en buffer y posteriormente la reacción se detuvo con $0,5 \mathrm{ml}$ de ácido tricloroacético (TCA) 20\% (p/v). En el tratamiento control, el TCA fue adicionado previo al sustrato. Posteriormente, todos los tubos fueron centrifugados por 5 min a $10.000 \mathrm{~g}$. La absorbancia de los sobrenadantes se midió a $280 \mathrm{~nm}$. Todos los ensayos fueron realizados por triplicado. La actividad proteásica total se expresó en unidades de actividad por mililitro de extracto proteico $\left(\mathrm{U} \mathrm{ml}^{-1}\right), \mathrm{y}$ fue calculada de la siguiente manera:

- para proteasas ácidas

$\mathrm{U} \mathrm{ml}^{-1}=\frac{\mathrm{Abs} 280 \mathrm{~nm} \times \mathrm{Vt}}{0,051 \times \mathrm{t} \times \mathrm{Ve}}$

- para proteasas alcalinas

$\mathrm{U} \mathrm{ml}^{-1}=\frac{\mathrm{Abs} 360 \mathrm{~nm} \times \mathrm{Vt}}{\mathrm{t} \times \mathrm{Ve}}$ 
donde:

0,051 = coeficiente de extinción molar de la Tirosina a $280 \mathrm{~nm}$ en la ecuación de proteasas ácidas $\mathrm{y} \mathrm{Vt}=$ volumen final de reacción $(\mathrm{ml}) ; \mathrm{t}=$ tiempo de reacción (minutos); $\mathrm{Ve}=$ volumen del extracto enzimático evaluado (ml) para ambas ecuaciones.

A su vez, la actividad proteásica específica fue calculada considerando el contenido proteico de los extractos, y se expresó como unidades de actividad por miligramo de proteína soluble $\left(\mathrm{U} \mathrm{mg}^{-1}\right)$. La misma se calcula de la siguiente manera:

- para proteasas ácidas

$\mathrm{U} \mathrm{mg}^{-1}=\frac{\mathrm{Abs} 280 \mathrm{~nm} \times \mathrm{Vt}}{0,051 \times \mathrm{t} \times \mathrm{Ve} \times \mathrm{mg} \text { proteína }}$

- para proteasas alcalinas

$\mathrm{Umg}^{-1}=\frac{\text { Abs } 360 \mathrm{~nm} \times \mathrm{Vt}}{\mathrm{t} \times \mathrm{Ve} \times \mathrm{mg} \text { proteína }}$

La actividad de proteasas alcalinas fue evaluada en los extractos enzimáticos de acuerdo con García-Carreño (1992a), utilizando azocaseína (Sigma A2765) como sustrato al 0,5\% (p/v) en solución buffer Tris-HCl, $50 \mathrm{mM}$ pH 8. Cada tubo de reacción fue preparado con $10 \mu \mathrm{l}$ de extracto enzimático mezclados con $500 \mu \mathrm{l}$ de sustrato y $500 \mu \mathrm{l}$ de solución buffer, la mezcla se incubó durante $10 \mathrm{~min}$ a $25{ }^{\circ} \mathrm{C}$. La reacción fue detenida adicionando $500 \mu 1$ de TCA $20 \%(\mathrm{p} / \mathrm{v})$ y enfriando en hielo por $10 \mathrm{~min}$. En los tubos control, el TCA se agregó luego del extracto enzimático y antes del sustrato proteico, con el fin de inactivar la enzima y evitar su reacción. Luego, los tubos fueron centrifugados por $5 \mathrm{~min}$ a $10.000 \mathrm{~g}$. Todos los ensayos fueron realizados por triplicado. La absorbancia de los sobrenadantes fue medida a $360 \mathrm{~nm}$.

Se relativizó la actividad de proteasas acidas $\mathrm{y}$ alcalinas de ambos extractos enzimáticos (tra- tados y sin tratar con MBS), considerando como $100 \%$ a la actividad registrada en los langostinos sin tratar. A su vez, se estimó el rendimiento enzimático como la actividad proteásica por kilogramo de cabezas de langostino $\left(\mathrm{U} \mathrm{kg}^{-1}\right)$ de la siguiente manera:

- actividad proteásica ácida total por kilogramo de cabezas

$\mathrm{U} \mathrm{kg}^{-1}=\left(\frac{\mathrm{Abs} 280 \mathrm{~nm} \times 1}{0,051 \times \mathrm{t} \times \mathrm{Ve} \times \mathrm{Me}}\right)$

- actividad proteásica alcalina total por kilogramo de cabezas

$\mathrm{Ukg}^{-1}=\left(\frac{\mathrm{Abs} 360 \mathrm{~nm} \times 1}{\mathrm{t} \times \mathrm{Ve} \times \mathrm{Me}}\right)$

donde:

$\mathrm{Me}=$ masa de tejido $(\mathrm{kg})$

\section{Efecto del metabisulfito sobre la composición enzimática de los extractos}

Para evaluar el efecto del preservante sobre la composición de enzimas activas de los extractos enzimáticos de langostino, se utilizaron inhibidores específicos. Se identificó el porcentaje de inhibición de actividad de proteasas aspárticas, cisteínicas, serínicas y tripsina utilizando los inhibidores Pepstatina A, E 64, SBTI (Soybean Trypsin Inhibitor) y TLCK (N- $\alpha$-tosyl-L-lysylchloromethyl-ketone) respectivamente.

Para evaluar el efecto del MBS sobre composición de proteasas ácidas, todos los extractos enzimáticos (tratados y sin tratar con MBS) fueron incubados con Pepstatina A 1,45 mM (Sigma P5318) como inhibidor de proteasas aspárticas y E $641 \mathrm{mM}$ (Sigma E3132) como inhibidor de proteasas cisteínicas (Garcia-Carreño 1992b). Soluciones de $10 \mu \mathrm{l}$ de cada inhibidor fueron mezcladas por separado con los extractos enzi- 
máticos $(10 \mu \mathrm{l})$ e incubadas por $60 \mathrm{~min}$ a $25^{\circ} \mathrm{C}$ a $\mathrm{pH}$ 3. Se tomaron como controles de inhibición de actividad a los extractos enzimáticos sin presencia de inhibidor. Posteriormente se evaluó la actividad enzimática según la metodología descrita previamente (Anson 1938). Los ensayos fueron realizados por triplicado. El grado de inhibición de actividad fue calculado como porcentaje relativo considerando actividad del $100 \%$ a la registrada en cada extracto sin inhibidor y expresado como porcentaje promedio para cada tratamiento. Un mayor grado de inhibición por parte del inhibidor específico de la enzima indica una mayor predominancia de esta enzima activa sobre la actividad enzimática total ( $\sin$ inhibidor) de los extractos.

Para evaluar el efecto del MBS sobre la composición de proteasas alcalinas todos los extractos enzimáticos (tratados y sin tratar con MBS) fueron incubados con SBTI $250 \mu \mathrm{M}$ (Fluka 93618) como inhibidor de proteasas del tipo serino y TLCK $10 \mathrm{mM}$ (Fluka 90182) en $1 \mathrm{mM}$ $\mathrm{HCl}$ pH 3 como inhibidor de tripsina (GarciaCarreño 1992b). Soluciones de $10 \mu 1$ de cada inhibidor fueron mezcladas por separado con los extractos enzimáticos $(10 \mu 1)$ e incubadas por 60 min a $25^{\circ} \mathrm{C}$ a pH 8 . Se tomaron como controles de inhibición de actividad a los extractos enzimaticos sin presencia de inhibidor. Posteriormente se evaluó la actividad enzimática según la metodología descrita previamente (GarcíaCarreño 1992a). Los resultados se expresaron como se describió para la inhibición de las proteasas ácidas.

\section{Análisis estadísticos}

Para comparar la actividad total, la actividad específica y el rendimiento por kilogramo tanto de proteasas ácidas como alcalinas (en langostinos con y sin MBS) se realizó el análisis de varianzas (ANOVA) de una vía, luego de aplicar los test de normalidad y homogeneidad de varianzas. Las diferencias significativas fueron consideradas con un valor de $\mathrm{P} \leq 0,05$. Cuando se encontraron diferencias, se aplicó el test de comparaciones múltiples de Tukey-Kramer. Los análisis se realizaron mediante el software estadístico NCSS 2007.

\section{RESULTADOS}

La actividad total, la actividad específica y el rendimiento por kilogramo de proteasas ácidas y alcalinas fue significativamente menor en extractos enzimáticos de langostinos tratados con MBS respecto de los ejemplares sin tratar ( $\mathrm{P}$ $<0.05$; Tabla 1; Figura 1). Los valores de actividad específica tanto para proteasas acidas como alcalinas mantienen las mismas diferencias significativas que los valores registrados para actividad total. Con respecto al rendimiento por kilogramo, la actividad de proteasas acidas se vio más afectada por el tratamiento con MBS $\left(5.610,37 \pm 3.247,14 \mathrm{U} \mathrm{kg}^{-1}\right.$ versus 807,41 \pm $\left.201,74 \mathrm{U} \mathrm{kg}^{-1}\right)$ que las proteasas alcalinas $\left(6.158,63 \pm 872,58 \mathrm{U} \mathrm{kg}^{-1}\right.$ versus 2.056,78 \pm $528,53 \mathrm{U} \mathrm{kg}^{-1}$ ) (Tabla 1).

En cuanto a la composición de enzimas activas y al efecto del preservante, se evidenciaron cambios en la actividad de los distintos tipos de extractos enzimáticos (Tabla 2). El tratamiento de los extractos enzimáticos con MBS afecta negativamente la actividad de las enzimas aspárticas dado que el porcentaje de inhibición en presencia de Pepstatina A es menor con respecto a lo observado en el extracto proteico sin tratar con MBS. Por otra parte, el porcentaje de inhibición de las enzimas cisteínicas en presencia del inhibidor enzimático E64 en el extracto proteico tratado con MBS es mayor que en el extracto sin tratar, lo cual representa una mayor participación en la actividad en los extractos tratados con MBS. En cuanto a las proteasas alcalinas, no se observaron cambios en la actividad de enzimas serínicas en su totalidad; sin embargo, las tripsi- 
Tabla 1. Actividad total, actividad específica y rendimiento de proteasas acidas y alcalinas de extractos enzimáticos de Pleoticus muelleri tratados y sin tratar con MBS. Los valores se expresan como media y desvió estándar. Distintas letras (a y b) indican diferencias estadísticamente significativas para cada tipo de actividad enzimática $(\mathrm{P}<0,05)$.

Table 1. Total activity, specific activity and performance of acid and alkaline proteases of enzymatic extracts of Pleoticus muelleri treated and without treatment with MBS. Values are expressed as mean and standard deviation. Different letters (a and b) indicate statistically significant differences for each type of enzyme activity $(P<0.05)$.

\begin{tabular}{|c|c|c|c|c|}
\hline & \multicolumn{2}{|c|}{ Proteasas ácidas } & \multicolumn{2}{|c|}{ Proteasas alcalinas } \\
\hline & Sin MBS & Con MBS & Sin MBS & Con MBS \\
\hline Actividad total $\left(\mathrm{U} \mathrm{m}^{-1}\right)$ & $1,40 \pm 0,812^{\mathrm{a}}$ & $0,20 \pm 0,050^{\mathrm{b}}$ & $1,54 \pm 0,218^{\mathrm{a}}$ & $0,51 \pm 0,132^{\mathrm{b}}$ \\
\hline $\begin{array}{l}\text { Actividad específica } \\
\left(\mathrm{U} \mathrm{mg}^{-1}\right)\end{array}$ & $0,25 \pm 0,146^{\mathrm{a}}$ & $0,01 \pm 0,003^{b}$ & $0,28 \pm 0,039^{a}$ & $0,03 \pm 0,008^{b}$ \\
\hline $\begin{array}{l}\text { Rendimiento por } \\
\text { kilogramo }\left(\mathrm{U} \mathrm{kg}^{-1}\right)\end{array}$ & $5.610,37 \pm 3.247,138^{a}$ & $807,41 \pm 201,737^{\mathrm{b}}$ & $6.158,63 \pm 872,580^{\mathrm{a}}$ & $2.056,78 \pm 528,526^{\mathrm{b}}$ \\
\hline
\end{tabular}

Tabla 2. Porcentaje de inhibición de proteasas de extractos enzimáticos de Pleoticus muelleri tratados y sin tratar con MBS con el uso de diferentes inhibidores enzimáticos.

Table 2. Inhibition percentage of proteases of the enzyme extracts of Pleoticus muelleri treated and without treatment with the use of different enzyme inhibitors.

\begin{tabular}{|c|c|c|c|}
\hline \multirow[b]{2}{*}{ Proteasas } & \multirow[b]{2}{*}{$\begin{array}{l}\text { Inhibidor } \\
\text { enzimático }\end{array}$} & \multicolumn{2}{|c|}{ Inhibición (\%) } \\
\hline & & Sin MBS & Con MBS \\
\hline
\end{tabular}

Ácidas

$\begin{array}{llll}\text { Aspárticas } & \text { Pepstatina A } & 87,8 & 58,6 \\ \text { Cisteínicas } & \text { E64 } & 58,6 & 80,0\end{array}$

Alcalinas

\begin{tabular}{llll} 
Serínicas & SBTI & 73,2 & 70,8 \\
Tripsina & TLCK & 31,6 & 77,8 \\
\hline
\end{tabular}

nas presentan un mayor porcentaje de participación en la actividad total en los extractos tratados con MBS, ya que el porcentaje de inhibición en presencia del inhibidor de tripsinas TLCK es mayor con respecto a los extractos sin tratar.

\section{DISCUSIÓN}

Hasta el momento existe poca información con respecto al uso de preservantes alimentarios y su impacto en la integridad de enzimas proteásicas de langostinos. Rodríguez-Casariego et al. (2014) propusieron que el uso de inhibidores de la PFO podría resultar beneficioso para preservar la actividad de las enzimas proteásicas de crustáceos. Estos autores sugieren que las proteasas presentan cambios conformacionales que disminuyen su capacidad catalítica debido a la acción de la PFO, enzima que produce especies reactivas de oxígeno y quinonas. Sin embargo, en este trabajo se observó una disminución de aproximadamente el $80 \%$ en la actividad proteásica de los langostinos tratados con MBS, por lo cual el conservante afectó negativamente la actividad de proteasas ácidas y alcalinas. Estos resultados coinciden con los obtenidos por Lalithapriya et al. (2019) quienes determinaron mediante análisis calorimétrico la reducción de la actividad de las enzimas autolíticas endógenas de camarón por efecto del bisulfito de sodio. Por la naturaleza nucleofílica del 


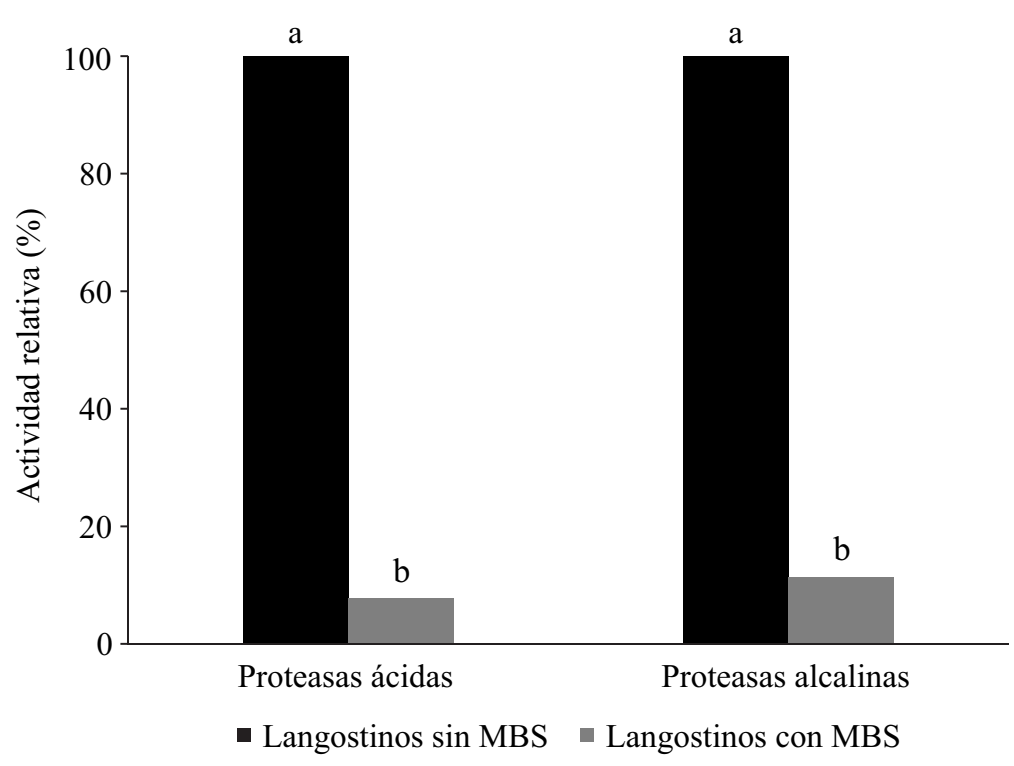

Figura 1. Actividad relativa de proteasas ácidas y alcalinas en Pleoticus muelleri tratados y sin tratar con metabisulfito de sodio (MBS). Distintas letras (a y b) indican diferencias estadísticamente significativas para cada tipo de actividad enzimática $(\mathrm{P}<0,05)$.

Figure 1. Relative activity of acidic and alkaline proteases in Pleoticus muelleri treated and without treatment with sodium metabisulfite (MBS). Different letters ( $a$ and $b$ ) indicate statistically significant differences for each type of enzyme activity $(P<0.05)$.

bisulfito liberado durante las reacciones que ocurren en los langostinos tratados, este compuesto es capaz de unirse a otros grupos funcionales además de las quinonas, pudiendo generar la inhibición no solo de la PFO sino también de diferentes enzimas (Thein y Michalke 1988).

El rendimiento de actividad enzimática estimado por kilogramo de cabezas fue significativamente mayor para langostinos sin MBS tanto para proteasas acidas como alcalinas $(5.610,37 \pm$ $3.247,14 \mathrm{U} \mathrm{kg}^{-1}$ y $6.158,63 \pm 872,58 \mathrm{U} \mathrm{kg}^{-1}$ respectivamente). Sin embargo, a pesar del efecto negativo del tratamiento con MBS sobre las enzimas de langostino, los valores de rendimiento por kilogramo de cabezas obtenidos $(807,41 \pm$ $201,74 \mathrm{U} \mathrm{kg}^{-1}$ para proteasas acidas y $2.056,78 \pm$ $528,53 \mathrm{U} \mathrm{kg}^{-1}$ para proteasas alcalinas) indican que las mismas representan una interesante fuente de enzimas para elaborar coagulantes lácteos, hidrolizados proteicos y aditivos enzimáticos para alimentación animal (Pereira y Fernández-
Giménez 2016, 2017; Rodríguez et al. 2017, 2018, 2021; Pereira et al. 2018, 2020a, 2020b, 2021).

El tratamiento de los langostinos con MBS registró cambios en la composición de enzimas activas en los extractos enzimáticos de esta especie. Se redujo la actividad de las enzimas aspárticas, lo cual indicaría que este preservante además de inhibir la PFO, también actuaría como inhibidor de estas enzimas. Según Lupano (2013) el bisulfito actúa reaccionando con los grupos carbonilo, las bases de Schiff, y los dobles enlaces $\mathrm{C}=\mathrm{C}$, dando sulfonatos muy estables. Debido a la presencia de grupos carbonilos en las enzimas aspárticas, posiblemente el mecanismo de acción de MBS sea similar al que ocurre con otros inhibidores aspárticos, los cuales se unen al grupo carbonilo del residuo aminoacídico del sitio activo de la enzima mediante puentes de hidrógeno, generando su inactivación (Pearl 1987). Por otro lado, se observó que los extractos enzimáticos de langostinos tratados con MBS registraron una 
mayor actividad de enzimas cisteínicas. Según Whitehead et al. (2001) y LaVoie et al. (2005) durante el proceso de melanosis, las especies reactivas generadas reaccionan con los residuos de cisteína, por lo cual el uso del MBS pudo haber inhibido la producción de estos compuestos reactivos, ejerciendo un efecto positivo sobre la actividad de estas enzimas proteásicas.

En cuanto a las proteasas alcalinas, no se observaron cambios en la composición de enzimas serínicas en su totalidad, mientras que se registró un alto porcentaje de inhibición de actividad de tripsina. Esto indicaría que a pesar de que la actividad de las enzimas alcalinas se vio afectada negativamente por el uso del preservante, las tripsinas se encuentran activas en mayor proporción en la composición enzimática total. Por otro lado, la abrupta reducción de la actividad alcalina en los langostinos tratados con MBS podría deberse a que este compuesto ha provocado la ruptura y/o reordenamiento de enlaces disulfuro en las enzimas provocando su inactivación (Zhang y Sun 2008; Guerrero-Olazarán et al. 2019). Esto último concuerda con los resultados obtenidos por Friedman y Gumbmann (1986), quienes detectaron una reducción en la actividad inhibidora de tripsinas de harina de soja en presencia de sulfito de sodio.

Las evaluaciones realizadas en este trabajo demuestran que el tratamiento de $P$. muelleri con MBS tiene un efecto negativo sobre la actividad de enzimas ácidas y alcalinas, además de presentar cambios en la composición de enzimas activas en los extractos enzimáticos de esta especie. Sin embargo, a pesar de la drástica reducción de la actividad enzimática, estos extractos constituyen una buena fuente de proteasas considerando los importantes volúmenes de cabezas de langostinos desechados, los cuales presentan altos valores de actividad enzimática por kilogramo. Además, estos extractos proveen principalmente actividad de enzimas cisteínicas y serínicas, mientras que la actividad de enzimas aspárticas se ve reducida. Sería importante evaluar en trabajos futuros el uso y dosis de otros preservantes inhibidores de la melanización que mantengan la integridad y composición de las proteasas del langostino, y de esta manera lograr un aprovechamiento más eficiente de estos residuos a la hora de elaborar subproductos de valor agregado.

\section{AGRADECIMIENTOS}

Los autores agradecen al Consejo Nacional de Investigaciones Científicas y Técnicas (CONICET) por subvencionar la formación doctoral de la Lic. Clara Liebana, y a la Universidad Nacional de Mar del Plata (UNMdP) por otorgar los siguientes subsidios por los cuales este trabajo fue llevado a cabo: EXA 874-18, 875-18 y 968/20.

\section{REFERENCIAS}

ANSON ML. 1938. The estimation of pepsin, trypsin, papain, and cathepsin with hemoglobin. J Gen Physiol. 22: 79-89.

Bovcon ND, Góngora ME, Marinao C, GonZÁlez Zevallos D. 2013. Composición de las capturas y descartes generados en la pesca de merluza común Merluccius hubbsi y langostino patagónico Pleoticus muelleri: un caso de estudio en la flota fresquera de altura del Golfo San Jorge, Chubut, Argentina. Rev Biol Mar Ocean. 48: 303-319.

BRADFORD MM. 1976. A rapid and sensitive method for the quantitation of microgram quantities of protein utilizing the principle of protein-dye binding. Anal Biochem. 72: 248-254.

CARranza-Espinal EO. 2014. Comparación de tres metodologías para la captación de sulfitos en camarones tratados con metabisulfito de sodio. Rev Cienc Tecnol. 14: 62-76.

DíAz-LóPEz MI, MARTÍNEZ-DíAz T, MartínezMoya M, Montero-García M, Gómez-Gui- 
LLÉN J, ZAMORANO-RODRÍGUEZ Y MARTÍNEZ Álvarez O. 2003. Estudios de los agentes conservantes e inhibidores de la melanosis en crustáceos. Andalucía: Junta de Andalucía Consejería de Agricultura y Pesca. 188 p.

Ferrer OJ, Otwell S, Marshall MR. 1989. Effect of bisulfite on lobster shell phenoloxidase. J Food Sci. 54 (2): 478-480.

FRIEDMAN M, GUMBMANN MR. 1986. Nutritional improvement of soy flour through inactivation of trypsin inhibitors by sodium sulfite. J Food Sci. 51: 1239.

GARCÍA-CARREÑo FL. 1992a. The digestive proteases of langostilla Pleuronco desplanipes, Decapoda: Their partial characterization and the effect of fed on their composition. Comp Biochem Physiol. 103B: 575-578.

García-CARREÑo FL. 1992b. Protease inhibition in theory and practice. Biotech Educ. 3: 14550.

Góngora Me, González-Zevallos D, PettoveLlo A, Mendia L. 2012. Caracterización de las principales pesquerías del golfo San Jorge Patagonia, Argentina. Lat Am J Aquat Res. 40 (1): 1-11.

Guerrero-Olazarán M, Castillo-Galván M, Gallegos-López JA, Fuentes-Garibay JA, Viader-Salvadó JM. 2019. Biochemical characterization of recombinant Penaeus vannamei trypsinogen. Comp Biochem Physiol B, Biochem Mol Biol. 238: 110337.

Lalithapriya U, Mariajenita P, RenUKa V, SUdHARSAN K, KaRTHIKEYAN S, Sivarajan M, Murugan D, Sukumar M. 2019. Investigation of natural extracts and sodium bisulfite impact on thermal signals and physicochemical compositions of Litopenaeus vannamei during chilled storage. J Aquat Food Prod Technol. 28 (6): 1-15.

LaVoie MJ, Ostaszewski BL, Weihofen A, Schlossmacher MG, Selkoe DJ. 2005. Dopamine covalently modifies and functionally inactivates parkin. Nat Med. 11 (11): 1214-1221.
LuPANO CE. 2013. Modificaciones de componentes de los alimentos: cambios químicos y bioquímicos por procesamiento y almacenamiento. La Plata: Editorial de la Universidad de La Plata (EDULP). 218 p.

[MAGyP] Ministerio de Agricultura, GanaDERÍA Y PESCA. 2021. Desembarques. https:// www.magyp.gob.ar/sitio/areas/pesca_mariti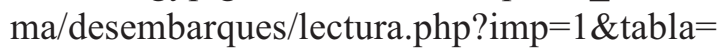 especie_flota_2020.

Moriondo Danovaro P, de la Garza J. 2019. Pesquería de langostino (Pleoticus muelleri). Resumen de la información biológica-pesquera reportada por los observadores a bordo en aguas de jurisdicción nacional. Temporada 2018. Inf Téc Of INIDEP No 9/2019. 14 p.

Otell SLG. 2010. Farm raised shrimp, good aquaculture practices for product quality and safety. Florida: Aquat Food Prod Progr. 71 p.

PEARL LH. 1987. The catalytic mechanism of aspartic proteinases. FEBS Lett. 214 (1): 8-12.

Pereira, NDLÁ, Fangio MF, Rodriguez Ye, Bonadero MC, Harán NS, FernándezGiMÉnEZ AV. 2021. Characterization of liquid protein hydrolysates shrimp industry waste: analysis of antioxidant and microbiological activity, and shelf life of final product. J Food Process Preserv. 00: e15526.

Pereira NDLÁ, Fangio MF, Rodriguez Ye, GARBARI DF, FERNÁNDEZ-GimÉnEZ AV. 2020a. Obtención de hidrolizados proteicos a partir de desechos de la industria pesquera. En: SARAIVA PinheIro MS, editor. Oceanografía: develando la belleza, los misterios y los desafíos del mar. Curitiba: Editora Artemis. p. 99110.

PEREIRA NDLÁ, FERnÁNDEZ-GimÉnEz AV. 2016. Revalorización de subproductos de la pesca y su estado actual en Argentina y otros países de América Latina. Düsseldorf: Editorial Académica Española. 72 p.

PEREIRA NDLÁ, FERnÁNDEZ-GIMÉNEZ AV. 2017. Exogenous enzymes in dairy technology: acidic proteases from processing discards of 
shrimp Pleoticus muelleri and their use as milk-clotting enzymes for cheese manufacture. Int J Food Sci Technol. 52: 341-347.

PEREIRA NDLÁ, HarÁN NS, Rodriguez YE, FernándeZ-GimÉnEZ AV. 2020b. Propuesta de un desarrollo industrial sostenible post covid19: coagulante lácteo a partir de la utilización de restos del procesamiento de especies pesqueras comerciales mediante tecnología verde. Latvia: Editorial Académica Española. $76 \mathrm{p}$.

Pereira NDLÁ, Rodriguez Ye, Garbari D. 2018. Técnicas de obtención y utilización de enzimas de interés industrial provenientes de desechos del procesamiento del langostino argentino comercial Pleoticus muelleri. Latvia: Editorial Académica Española.

Pettovello AD, Boschi EE. 2016. La fauna acompañante del langostino patagónico (Pleoticus muelleri) en el Golfo San Jorge y adyacencias: análisis de alternativas de manejo. En: Boschi EE, editor. El Mar Argentino y sus recursos pesqueros. Tomo 6. Los crustáceos de interés pesquero y otras especies relevantes en los ecosistemas marinos. Mar del Plata: Instituto Nacional de Investigación y Desarro1lo Pesquero (INIDEP). p. 89-94.

Rodríguez YE, Laitano MV, Pereira NA, López-Zavala AA, Haran NS, FernándeZGIMÉNEZ AV. 2018. Exogenous enzymes in aquaculture: alginate and alginate-bentonite microcapsules for the intestinal delivery of shrimp proteases to Nile tilapia. Aquaculture. 490: 5-43.
Rodríguez Ye, Pereira NA, Haran NS, Mallo JC, FernándeZ-GimÉnez AV. 2017. A new approach to fishery waste revalorization to enhance Nile tilapia (Oreochromis niloticus) digestion process. Aquacult Nutr. 23 (6): 1351-1361.

Rodríguez Ye, Pereira NA, Laitano MV, Moreno P, FERnÁndeZ-Giménez AV. 2021. Exogenous proteases from seafood processing waste as functional additives in rainbow trout aquaculture. Aquacult Res. 52 (9): 4350-4361. doi:10.1111/are.15272

Rodríguez-Casariego J, Perdomo-Morales R, Perera E. 2014. Purificación de isoformas de proteasas tipo tripsina de crustáceos. Rev Invest Mar. 32: 1-8.

Thein M, Michalke W. 1988. Bisulfite interacts with binding sites of the auxin-transport inhibitor N-1-naphthylphthalamic acid. Planta. 176 (3): 343-350.

Whitehead RE, Ferrer JV, JaVitch JA, Justice, JB. 2001. Reaction of oxidized dopamine with endogenous cysteine residues in the human dopamine transporter. J Neurochem. 76: 12421251.

Xu ZH, Regenstein JM, Xie DD, Lu WJ, Ren XC, Yuan JJ, Mao LC. 2018. The oxidative stress and antioxidant responses of Litopenaeus vannamei to low temperature and air exposure. Fish Shellfish Immunol. 72: 564571.

ZHANG L, SUN XS. 2008. Effect of sodium bisulfite on properties of soybean glycinin. J Agr Food Chem. 56 (23): 11192-11197. 